\title{
A Theoretical Analysis of Optical Clock Extraction Using a Self-Pulsating Laser Diode
}

\author{
P. Rees, P. McEvoy, A. Valle, J. O’Gorman, S. Lynch, P. Landais, L. Pesquera, and J. Hegarty
}

\begin{abstract}
The potential for using inexpensive compact disc laser diodes as optical clock extraction elements in transparent networks has led to an increase in research into the dynamics of self-pulsating laser diodes. We use a rate-equation model to simulate the synchronization of the self-pulsating laser output pulses to a periodic optical signal. In particular, we investigate the time it takes for the laser to synchronize to the input signal and also, the time taken for the laser to unlock when the signal is removed. The effect of varying the power of the optical signal and the detuning of the input signal frequency relative to the laser's self-pulsation frequency are determined. Our results enable us to identify important issues which need to be addressed when a self-pulsating laser diode is used in a clock extraction subsystem. In particular, we find that the signal frequency and laser freerunning frequency must be as close as possible to minimize errors. Also, the higher the signal power the quicker the laser synchronizes to the signal, although we find that if the power becomes too large the laser can no longer lock, which would cause a significant increase in detection errors.
\end{abstract}

Index Terms-Optical clock extraction, semiconductor lasers, synchronization.

\section{INTRODUCTION}

$\mathbf{T}$ THE EVER-INCREASING demand for higher rates of data transfer has led to a significant research effort in the area of all-optical communications. It is widely believed that the low cost and availability of self-pulsating laser diodes means that these devices will play a major role in all-optical subsystems. The potential for optical clock extraction and clock distribution by the synchronization of a self-pulsating laser diode (SPLD) has already been demonstrated [1], [2]. In this paper, we have used a standard model for an SPLD to examine both the dynamics of the laser when an optical synchronizing signal is applied and how the synchronization is effected by changes in the optical input signal. This allows us to assess their potential for use in optical clock extraction

Manuscript received December 21, 1998; revised October 6, 1998. This work was supported by the British Council/Forbairt Joint Research Scheme and the Acciones Integradas Hispano-British Council no. HB1997-0214. The group at Instituto de Fisica de Cantabria would like to acknowledge financial support under the EU project CHRX-CT94-0594. The work of A. Valle and L. Pesquera was supported in part by CICYT Spain through Project TIC 95-0563-C05-01.

P. Rees is with the School of Electronic Engineering and Computer Systems, University of Wales Bangor, Bangor, Gwynedd LL57 1UT.

P. McEvoy, J. O'Gorman, S. Lynch, P. Landais, and J. Hegarty are with Optronics Ireland, Department of Physics, Trinity College Dublin, Dublin 2, Ireland.

A. Valle and L. Pesquera are with the Instituto de Fisica de Cantabria, CSIC-UC. Facultad de Ciencias, E-39005, Santander, Spain.

Publisher Item Identifier S 0018-9197(99)00850-7.

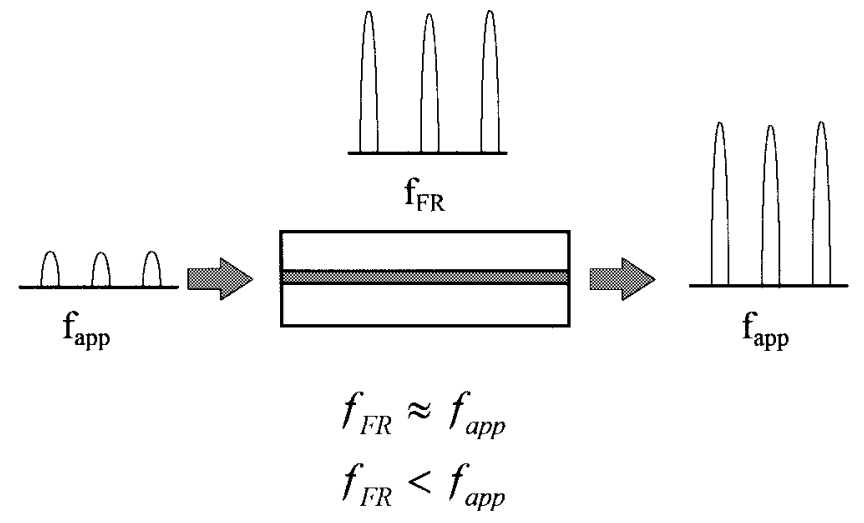

Fig. 1. A schematic showing the synchronization of an SPLD with an applied periodic optical signal.

subsystems and to optimize the laser operating conditions for low bit-error transmission.

This paper is the first of two related papers which investigate the use of self-pulsating laser diodes in optical clock extraction subsystems. In this paper, we outline the physical principles which effect the laser dynamics when a synchronizing external periodic optical input is applied to a laser diode. Our investigation enables us to make recommendations about the properties of the data signal relative to the laser output in order to reduce read errors due to a mistimed clock. The second paper [3] uses a similar model including noise sources to simulate the synchronization of a self-pulsating laser diode to a pseudorandom data signal in order to perform a full biterror calculation for the data signal using the clock generated by the laser diode. The results described in this paper are then used to minimize the bit-error rate in the system.

In order to be able to read each data bit in an optical data signal, the receiver must know the precise time at which to detect if the signal is " 0 or " 1 ." The receiver must therefore produce a periodic signal of exactly the same frequency as the data stream which can trigger the detector. If we consider a periodic optical signal applied to a free-running SPLD, as shown in Fig. 1, then providing the power of the optical signal is sufficient and the difference between the applied $\left(f_{\text {app }}\right)$ and free-running $\left(f_{F R}\right)$ SPLD frequencies is not too great, the laser output will lock (or synchronize) to the applied signal frequency. In this paper, we investigate the dynamics of the output of the SPLD when the periodic input signal is both applied and removed, and also when the properties of the input signal are changed. This allows us to assess the possibility of synchronizing an SPLD to a received data stream and use the 
TABLE I

PARAMETERS USED IN SimULATIONS

\begin{tabular}{|c|c|c|}
\hline Parameter & $\begin{array}{l}\text { Parameter } \\
\text { Symbol }\end{array}$ & Value \\
\hline Bimolecular radiative coefficient & B & $3.0 \times 10^{-10} \mathrm{~cm}^{3} \mathrm{~s}^{-1}$ \\
\hline Coefficient of Auger recombination & $\mathrm{C}$ & $7 \times 10^{-29} \mathrm{~cm}^{6} \mathrm{~s}^{-1}$ \\
\hline Gain section fraction & $\xi_{1}$ & calculated as in ref. [4] \\
\hline Absorber section fraction & $\xi_{2}$ & calculated as in ref. [4] \\
\hline Volume of the gain section & $\mathrm{V}_{1}$ & calculated as in ref. [4] \\
\hline Volume of the absorber section & $\mathrm{V}_{2}$ & calculated as in ref. [4] \\
\hline Langevin Noise term & $F_{s}(t)$ & calculated as in ref. [8] \\
\hline Group velocity of light & $v_{\mathrm{g}}$ & $7.5 \times 10^{9} \mathrm{~cm} \mathrm{~s}^{-1}$ \\
\hline $\begin{array}{l}\text { Transparency carrier density in the gain } \\
\text { and absorber regions }\end{array}$ & $\mathrm{n}_{01}, \mathrm{n}_{02}$ & $1.2 \times 10^{18} \mathrm{~cm}^{-3}$ \\
\hline Non-radiative recombination coefficient & $A_{\mathrm{nr}}$ & $1 \times 10^{8} \mathrm{~s}^{-1}$ \\
\hline $\begin{array}{l}\text { Gain and Absorption saturation } \\
\text { parameters }\end{array}$ & $\varepsilon_{1}, \varepsilon_{2}$ & $5 \times 10^{18} \mathrm{~cm}^{3}$ \\
\hline Differential gain parameter & $\alpha_{1}$ & $2 \times 10^{-16} \mathrm{~cm}^{2}$ \\
\hline Differential absorption parameter & $\alpha_{2}$ & $6 \times 10^{-16} \mathrm{~cm}^{2}$ \\
\hline Spontaneous emission coupling factor & $\beta$ & $1 \times 10^{-5}$ \\
\hline Current density & $j_{1}$ & $\begin{array}{l}\text { varied to obtain free running self } \\
\text { pulsation frequency }\end{array}$ \\
\hline Round-trip losses & $\alpha_{c}$ & $1200 \mathrm{~cm}^{-1}$ \\
\hline Active region thickness & d & $6 \times 10^{-6} \mathrm{~cm}$ \\
\hline
\end{tabular}

synchronized output of the laser diode to generate a clock in order to trigger the decision-making process on each data bit.

\section{THEORY}

Self-pulsation occurs in compact disc (CD) lasers under normal dc biasing conditions due to saturable absorption [4]. The optical field in the electrically pumped active region of the laser spreads out and overlaps the neighboring unpumped regions which act as the distributed saturable absorber. Selfpulsation occurs when volumes of the pumped and unpumped regions are properly balanced. The photon and carrier dynamics of such a laser can be described using three rate equations [4]. Two equations govern the rate of change of the carrier density in the central active region $\left(n_{1}\right)$ and the neighboring absorbing regions $\left(n_{2}\right)$ while a third equation governs the rate of change of the photon number $(S)$ in the laser cavity

$$
\begin{aligned}
\frac{d n_{1}}{d t}= & \frac{j_{1}}{e d}-n_{1}\left(A_{\mathrm{nr}}+B n_{1}+C n_{1}^{2}\right) \\
& -\xi_{1} v_{g} g\left(n_{1}\right) S / V_{1}-\frac{n_{1}-n_{2}}{\tau_{12}} \\
\frac{d n_{2}}{d t}= & -n_{2}\left(A_{\mathrm{nr}}+B n_{2}+C n_{2}^{2}\right) \\
& +\xi_{2} v_{g} \alpha\left(n_{2}\right) S / V_{2}-\frac{n_{2}-n_{1}}{\tau_{21}} \\
\frac{d S}{d t}= & v_{g}\left[\xi_{1} g\left(n_{1}\right)-\xi_{2} \alpha\left(n_{2}\right)-\alpha_{c}\right] S \\
& +\beta B n_{1}^{2} V_{1}+F_{S}(t)+S_{\text {in }}
\end{aligned}
$$

The gain and absorption coefficients are given by

$$
g\left(n_{1}\right)=\frac{\alpha_{1}\left(n_{1}-n_{01}\right)}{1+\varepsilon_{1} S / V_{1}} \quad \alpha\left(n_{2}\right)=\frac{\alpha_{2}\left(n_{02}-n_{2}\right)}{1+\varepsilon_{2} S / V_{2}} .
$$

The symbols used in the above equations are described in Table I together with typical values used. In particular, $\xi_{1}$ and $\xi_{2}$ represent the proportion of the optical power in the gain section and the side absorbing regions, respectively, and $\tau_{12}$ and $\tau_{21}$ are the diffusion times between the active and passive sections. These four parameters are recalculated every time step as described in [4] in order to describe the time development of the self-pulsation. $S_{\text {in }}$ represents the perturbation to the photon number due to the input synchronizing pulse. We have previously used this kind of calculation to successfully predict the behavior of the optical synchronization of a $\mathrm{CD}$ self-pulsating laser diode [5].

\section{TIME TAKEN TO SynChronize}

We have simulated the synchronization of the SPLD by adding a periodic impulse term $S_{\text {in }}$ to the photon number rate equation of the model to represent the optical input signal [5]. For our example, the optical input signal was assumed to be a train of rectangular pulses at a frequency of $2 \mathrm{GHz}$ and a duty cycle of $50 \%$. The number of photons in the rectangular pulse is modified to give the required input power (which depends on the wavelength of the photons and the length of the optical pulse). When investigating the synchronization of an SPLD experimentally, it is extremely difficult to measure the increase in photon number in the cavity because the coupling of the light pulse outside the cavity to the optical field in the cavity is difficult to determine. Unfortunately, this makes a detailed comparison between theory and experiment practically impossible, and, in this paper, we attempt to show the trends in the properties of the synchronization of the laser and not absolute comparisons with experiment.

In Fig. 2, we show the effect of the application of the periodic optical signal (at pulse number 20) on the periodic output of the SPLD. We have plotted the instantaneous frequency of the self-pulsation $v_{\mathrm{sp}}$ versus the pulse number, the instantaneous frequency being the inverse of the time 


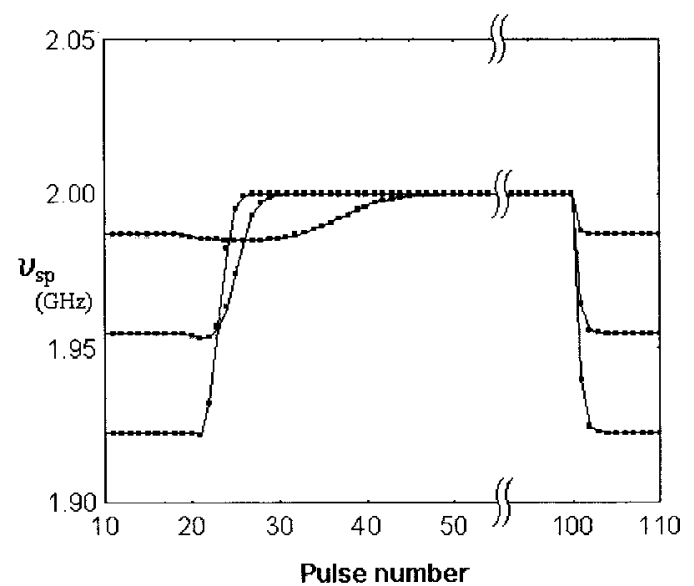

Fig. 2. The instantaneous self-pulsation frequency is shown versus pulse number for three free-running self-pulsation frequencies of $1.922,1.955$, and $1.987 \mathrm{GHz}$. The optical synchronization signal of $2 \mathrm{GHz}$ is applied at pulse 20 and removed at pulse 100 .

between successive pulses which allows us to monitor the laser output frequency on the pulse-to-pulse level. It is clear that when the optical signal is turned on the frequency of the SP is modified to the input frequency, as expected with synchronization. However, this change does not take place instantaneously, but over a number of pulse cycles. Curves are shown for initial free-running laser frequencies of 1.922, 1.955 , and $1.987 \mathrm{GHz}$; the larger the difference between the free-running and the synchronizing signal then the quicker the laser output locks to the applied signal. When the applied signal and laser output pulses are unsynchronized, the system cannot make a decision about any data bits received and so this gives an indication of how many redundant pulses must be sent before the data can be transmitted.

The frequency of the laser diode self-pulsation is varied by altering the bias current. The free-running self-pulsation frequency increases with increased bias current, in this case from $600 \mathrm{MHz}$ at threshold to $2.5 \mathrm{GHz}$ at twice threshold. We have found that the time to lock to the synchronizing frequency is also dependent on the initial phase difference between the input and output signal (as was the case for electrical synchronization [6]). In a clock extraction system, there would be no control over this phase difference and so the results presented in Fig. 2 are for cases where the initial phase difference gives the slowest locking times.

An example of the dependence of the time to lock to the synchronizing signal on the initial phase of the input pulse is given in Fig. 3. The locking time is shown as a function of the phase delay after the last output pulse in this case for a frequency detuning of $0.5 \%\left(0^{\circ}\right.$ corresponds to the start of the output pulse). Clearly there is a large difference in the locking time depending on the initial phase, and this result enables us to explain the increasing time to lock as the frequency detuning decreases. At certain times during the pulsation cycle the input pulse has a greater effect than at other times during the pulse period. For example the phase delay between 0 and $70^{\circ}$ corresponds to the output of the laser pulse, the large number of photons in the laser cavity means that the input pulse is only a very small perturbation to the system corresponding to a large

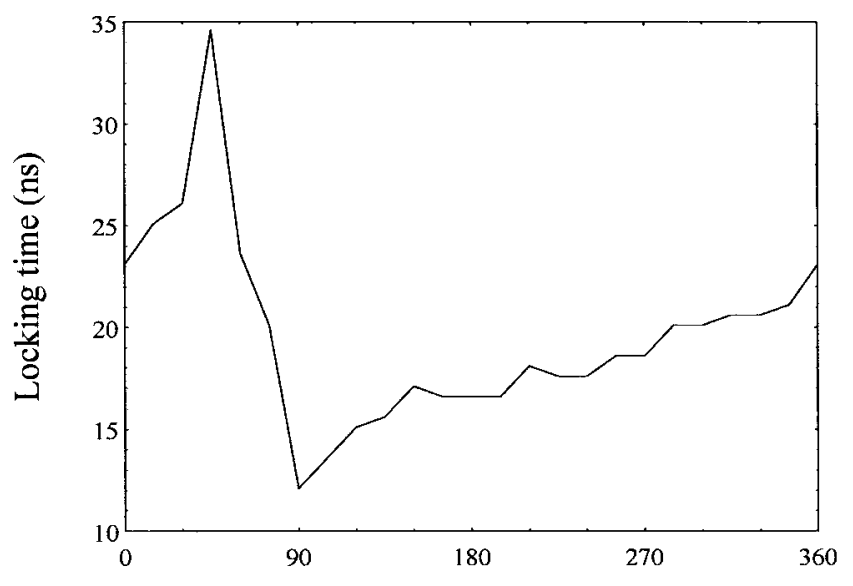

Phase delay after last pulse (degrees)

Fig. 3. The locking time is shown as a function of the phase delay after the last output pulse, in this case for a frequency detuning of $0.5 \%\left(0^{\circ}\right.$ corresponds to the start of the output pulse).

locking time. In between pulses, the input pulse causes a large perturbation due to the small number of photons in the cavity (spontaneous emission) resulting in quicker locking times.

To explain the increased locking time as the frequency detuning is decreased, we consider the initial input pulse entering the laser at a time in the output cycle where it has minimal perturbation (i.e., during or just after the previous output pulse). The next input pulse will enter the laser at a time during the output cycle which is determined by the frequency detuning between the input and output frequencies. The larger the frequency detuning the sooner an input pulse will be in a region where the perturbation is large and start the frequency synchronization process. Should the detuning be very small, a large number of input pulses will be required before synchronization can occur, resulting in a lengthy locking time.

Fig. 2 showed the locking dynamics for three different frequencies at one particular value of synchronizing signal power. In Fig. 4, we demonstrate the effect of the input power on the locking characteristics. The time for the SPLD to lock to the synchronizing optical signal of $2 \mathrm{GHz}$ is shown in Fig. 4 as a function of frequency detuning for three different signal powers. The higher the signal power then the larger the frequency range over which synchronization occurs and the shorter the locking times. For clock extraction, a reduction in the time taken for the free-running SPLD's to lock to the applied signal and also an ability to extract a clock from as broad a range of data rates as possible are both desirable. Therefore, our initial investigation indicates that the data signal should be as optically intense as possible to achieve quick locking over a range of frequency detunings. However, we will show in Section V that problems can occur when the synchronization signal becomes too powerful.

While the data consists of consecutive 1's, the laser remains synchronized to the data, but obviously when the synchronizing signal is removed the SPLD reverts back to its free-running state. The important issue is how long the synchronized selfpulsation can be used as a clock when the incoming signal is removed or how many " 0 " bits in the data stream can be 


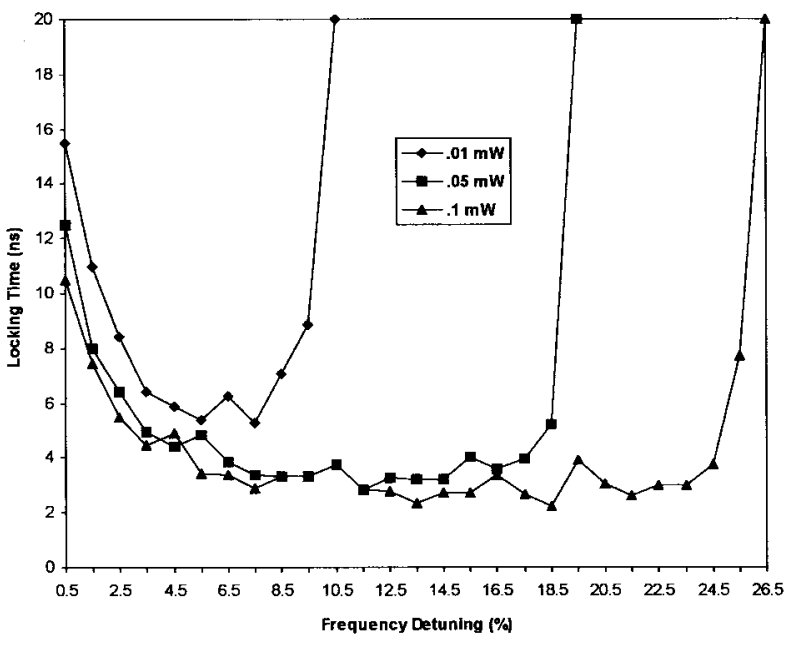

Fig. 4. The time for the SPLD to lock to the synchronizing signal is shown as a function of frequency detuning of the signal (relative to the free-running pulsation frequency). Examples for applied optical signal powers of 0.01 , 0.05 , and $0.1 \mathrm{~mW}$ are given.

accommodated before the clock signal drifts out of time with the input bits. Fig. 2 also shows the behavior of the SPLD when the synchronizing optical signal has been removed at pulse number 100. The important point to note is that the time for the laser to unlock is much faster than the time to lock (within 3-4 pulses) and is independent of the detuning. This result has been shown experimentally in a previous paper [7] and the effect verified using the same model as we employ in this study. This result means that after a few consecutive 0 's the SPLD would have reverted back to its free-running frequency and the clock would obtain a timing error with respect to the data signal. Initially, this would seem to suggest that SPLD's are unsuitable for clock extraction because the laser falls out of lock so quickly after the synchronizing signal is removed. This, however, is very misleading, as can be seen when we investigate (in Section VI) the phase difference between the applied optical signal and the SPLD output.

\section{PHASE}

Previously we have shown (both experimentally and theoretically) that a phase difference exists between an applied optical signal and the synchronized output of an SPLD [5]. This phase difference was shown to depend on the power and the frequency difference between the applied signal and the SPLD free-running output. In an optical clock extraction subsystem, the receiver would be required to compensate for this phase difference while reading the data. Ideally we want the decision-making process to coincide exactly with the midpoint of the rectangular data bit in time. The nature of a rectangular bit shape means we have a margin of error which depends on the length of the data bit. For a duty cycle of $50 \%$, the data pulse lasts for $180^{\circ}$ of the pulse cycle, and so for a perfect rectangular bit an error of $\pm 90^{\circ}$ in the timing can be accommodated. Of course, the data received would have degraded after transmission through optical fibers, etc., and this value would decrease for a realistic situation.

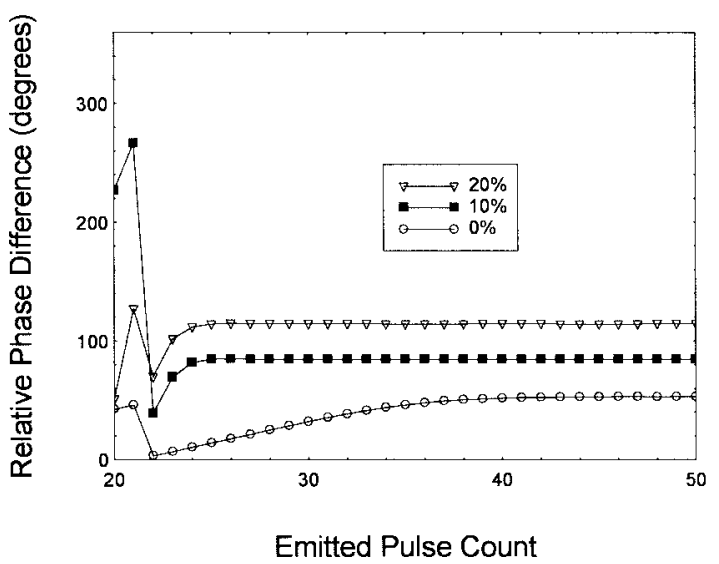

(a)

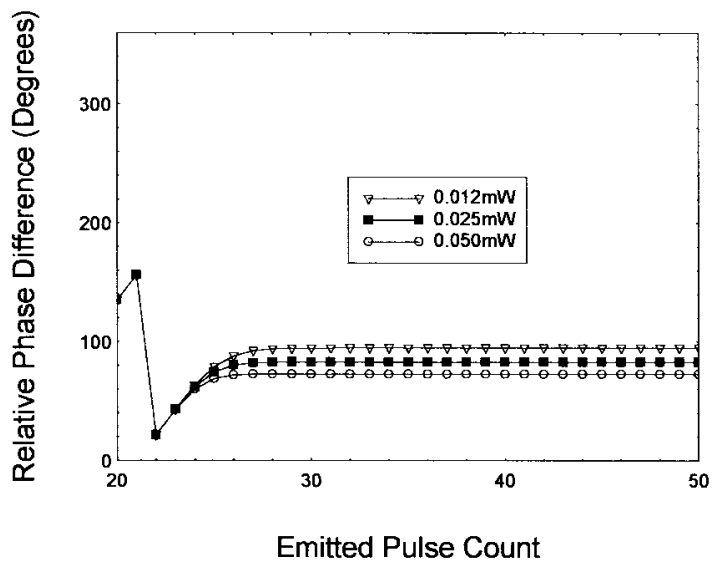

(b)

Fig. 5. (a) The instantaneous phase as a function of the applied pulse number for three different frequency-detuned applied signals (power $=0.025 \mathrm{~mW}$ ). The optical signal is applied at pulse number 20. (b) The instantaneous phase as a function of the applied pulse number for three different applied signal powers (detuning $=10 \%$ ). Again the optical signal is applied at pulse number 20 .

An important quantity for analyzing synchronization of SPLD's is the instantaneous phase difference. This is defined as the phase between an applied signal pulse and the next emitted pulse from the laser [5], given by

$\Delta \Phi_{D}=f_{\text {app }} \cdot 2 \pi \cdot($ time between signal and output pulse)

When the self-pulsating laser is synchronized to an applied signal, there is a fixed instantaneous phase difference, dependent on the detuning between the applied frequency $f_{\text {app }}$ and the free-running frequency [5]. Fig. 5(a) is a plot of the instantaneous phase as a function of the applied pulse number for three different frequency-detuned applied signals. With no noise in this calculation, the instantaneous phase remains constant, for all signals, once synchronization has been achieved. The value of the instantaneous phase increases with the detuning between the applied signal and free-running repetition frequencies. When the detuning is zero, we see the time to lock takes about 20 pulses, which is much longer than for nonzero detuning, as was seen in Fig. 2.

Fig. 5(b) shows the instantaneous phase as a function of the applied pulse number for three different applied signal powers. Clearly, the instantaneous phase when the laser is synchronized decreases with increasing applied power. A 
stronger signal pulse injected into the SP laser causes the synchronized pulse to be emitted earlier. The time difference between the extracted clock and the decision-making process can be optimized for one particular phase difference. If the power of the signal changes (which is highly likely in a transparent network where signals arrive via different paths) or if the frequency of the incoming signal changes, then the phase difference changes. This means the decisionmaking process is no longer in the center of the bit slot, significantly increasing the likelihood of errors. It is clear then that a detailed knowledge of how the power and frequency of the data signal arriving at the clock extraction element would vary is needed in order to compensate for the effect this has on the phase difference and, therefore, the timing of the data reading process. Previously, we have measured the variation of the phase difference between the optical synchronizing signal and the output of the SPLD (as a function of both power and frequency detuning), and our model's results showed excellent agreement with these experiments [5]. As mentioned earlier, exact comparison of experiment and theory was impossible because the signal coupling to the optical field in the cavity could not be measured. Our model successfully predicts the experimental frequency and power limits in which lock can be achieved [5] as well as the regions where lock cannot be obtained. Recently, this model has been used further to verify the synchronization limits for the cases of optical synchronization with frequency multiplication and division [7]. Most of the initial results of this paper would be very difficult to measure experimentally because we are studying timings on the pulse-to-pulse level where noise would play a major role (although we have verified the model using optical power spectrum measurements). Despite this, the success of this kind of model in predicting a large number of experimentally observed properties of an SPLD means we have a high level of confidence in its predictions.

As seen in Fig. 4, the higher the input signal power, the quicker the SPLD locks to the synchronizing signal. Therefore, it is to be expected that the signal power should be as high as possible for efficient clock extraction, however, we will show in the next section that problems can arise when the signal power becomes too large.

\section{EFFect of Signal Power}

Fig. 6 shows the instantaneous phase over 130 emitted pulses for the three extremes of applied optical power. The input optical pulses are applied after the tenth emitted pulse, at an initial phase of $360^{\circ}$. The lowest applied optical power, the dashed line, corresponds to a pulse peak power of $10 \mu \mathrm{W}$. The self-pulsating laser slowly synchronizes to the applied signal as seen by the instantaneous phase decreasing. Synchronization will be complete when the phase settles at a steady value. The second curve, the solid line, corresponds to an applied peak power of $25 \mu \mathrm{W}$. Here the self-pulsating laser synchronizes after about 30 applied pulses. The instantaneous phase remains constant, its value related to the applied optical power and the detuning between the repetition frequencies of the applied

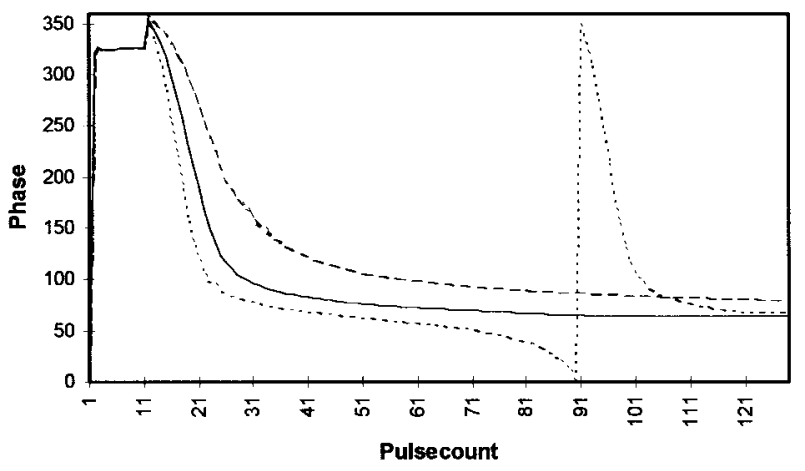

Fig. 6. The instantaneous phase over 100 emitted pulses for applied optical powers of $10 \mu \mathrm{W}$ (the dashed line), $25 \mu \mathrm{W}$ (the solid line), and $400 \mu \mathrm{W}$ (the dotted line). The input optical pulses are applied after the tenth emitted pulse, at an initial phase of $360^{\circ}$.

and free-running pulses, as shown in Fig. 5(a) and (b). The final curve, the dotted line, corresponds to an applied peak optical power of $400 \mu \mathrm{W}$. Here the self-pulsating laser seems to synchronize within a few pulses, but the instantaneous phase is not constant and slowly decreases, thereby showing that synchronization has not occurred. The phase decreases until the output pulse occurs "before" the signal pulse, which corresponds to $360^{\circ}$.

Previously, we have shown that the single-mode rateequation model will not allow the self-pulsating laser to lock to a signal frequency which is less than the self-pulsation frequency [5]. This causes the locking range to be very asymmetric about the signal frequency, and it also explains the reason for the phase oscillations. The stronger the applied optical signal power becomes, the larger the optical pumping effect, and therefore the larger the increase in the self-pulsation frequency of the laser. If the self-pulsation and the signal frequency are very close initially, a strong signal increases the self-pulsation frequency above the signal frequency, so the laser can no longer lock.

Fig. 7 helps to explain why we see phase oscillations when the laser cannot lock to the synchronizing signal. The time evolution of the signal and output pulses is shown together with the instantaneous phase difference between them when the input power is too large to synchronize the laser. The input pulse hastens the emission of the next output pulse, decreasing the phase between the signals, although the laser can no longer lock so the phase does not settle to a fixed value but continues to decrease. Once the instantaneous phase reaches zero degrees, two emitted pulses occur between the applied pulses, and the phase starts again at $360^{\circ}$. This process occurs periodically and appears as an oscillation of the instantaneous phase.

This phase oscillation reoccurs with a periodicity dependent on the applied optical power and the detuning between the applied and emitted pulse repetition frequencies, as shown in Fig. 8. The larger the detuning, the stronger the signal power required to increase the self-pulsation frequency above the signal frequency and cause a phase oscillation. Also shown in Fig. 8 is the periodicity with which the phase oscillations occur as a function of detuning for three different signal power levels. It is clear that the lower the power of the input signal 
(a)

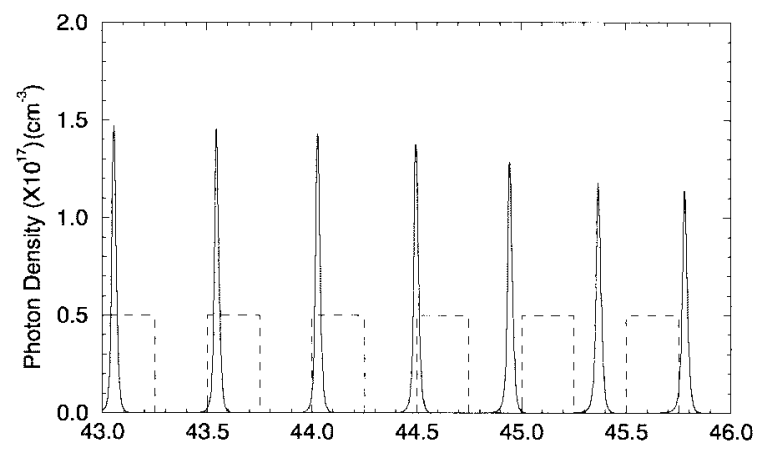

(b)

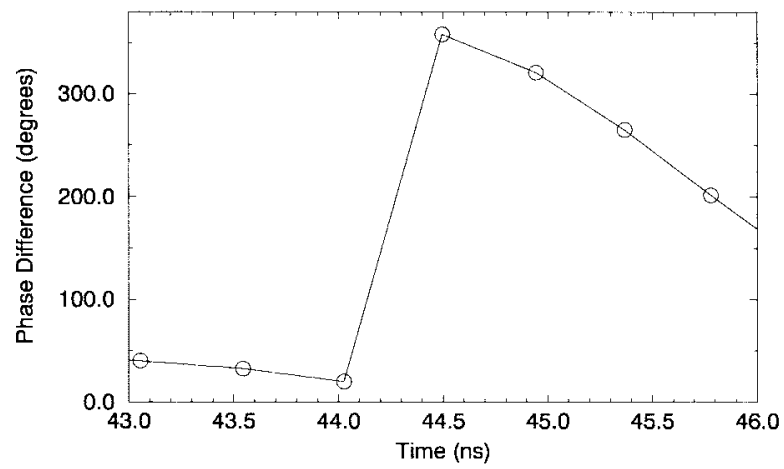

Fig. 7. (a) The dynamic evolution of the input signal (dashed lines) and the laser output pulses (solid lines) together with (b) the instantaneous phase difference between them for the case when the SPLD cannot lock to the input signal. The signal detuning is $1.05 \%$ and the input power is $0.07 \mathrm{~mW}$.

(a)

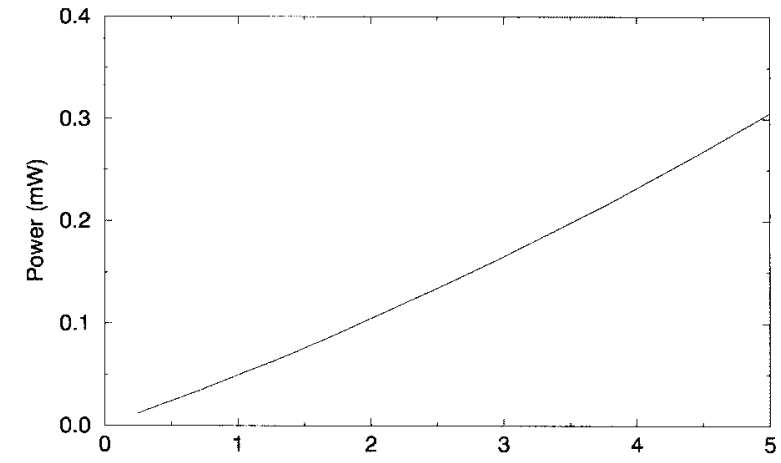

(b)

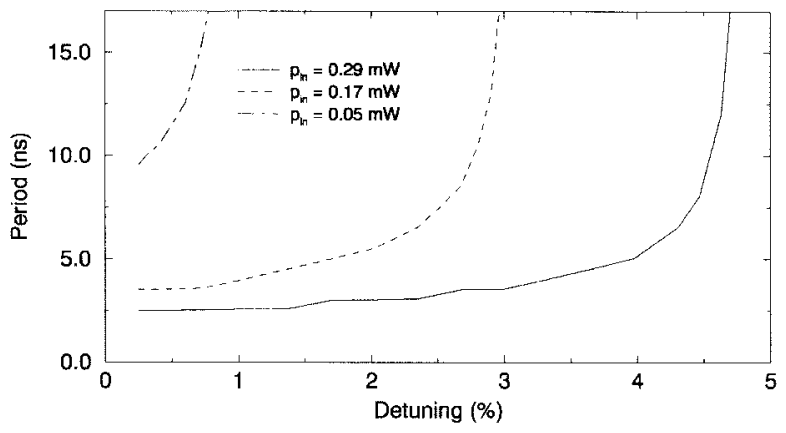

Fig. 8. (a) The power at which the phase oscillations appear together with (b) the period of these oscillations as a function of signal detuning.

then the longer the period of the phase oscillations for any detuning value. Again, we see that as we detune the selfpulsation frequency from the signal frequency the less likely is the occurrence of the phase oscillations.

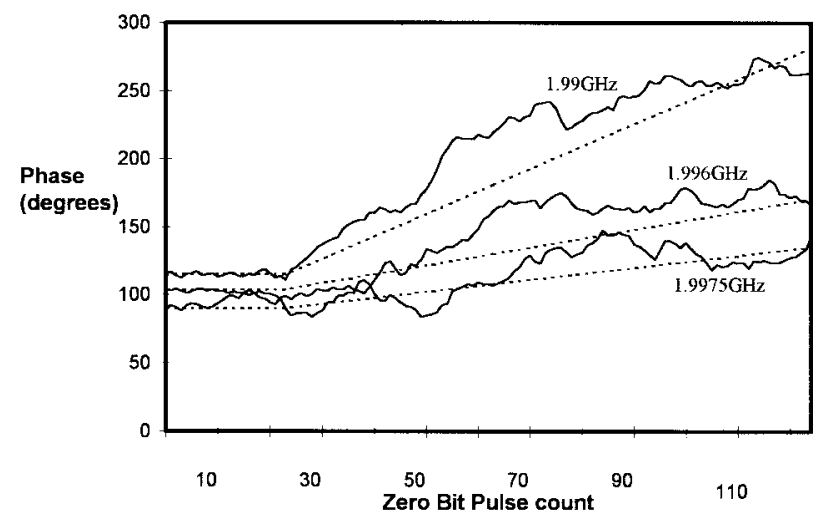

Fig. 9. The instantaneous phase difference between the input optical signal and the laser output after the synchronizing signal is removed at pulse number 25. Curves are shown for free-running frequencies of 1.99, 1.996, and 1.9975 $\mathrm{GHz}$. The solid and broken lines represent results with and without the inclusion of spontaneous emission noise, respectively.

When the laser can no longer synchronize to the signal (causing the phase oscillations to occur), it is impossible to use the laser to derive a clock signal; therefore, a detailed understanding of when this phenomena occurs is vital for clock extraction systems. When noise is included in the model, the successive decrease in the instantaneous phase which is observed when the free-running frequency is above the applied signal frequency is disrupted. This occurs because the random fluctuations due to noise can modify the instantaneous phase in such a way that it returns above its starting value, thus resetting the process. The phase oscillations therefore do not occur until a much stronger optical signal is applied, causing a successive reduction in phase, which occurs at a quicker rate than the rate at which the noise resets the process. The effect of noise on the loss of synchronization between the selfpulsating laser diode and the data signal is studied in detail in the second paper [3] where the full effect of this problem is related to a calculation of the bit error rate in such a system.

Previously, we saw that when the synchronizing optical signal is removed the SPLD reverts back to its free-running pulsation frequency within a couple of pulses. Initially this would suggest that the laser can no longer be used to successfully produce a clock for the read process. However, in the next section, we examine the relationship between the input signal and the laser output when the signal is removed in order to prove that under certain circumstances the unlocking of the laser is not too significant.

\section{LOSS OF PHASE}

Fig. 9 shows the instantaneous phase difference between the input optical signal and the laser output after the synchronizing signal is removed at pulse number 100. Curves are shown for free-running frequencies of 1.99, 1.996, and $1.9975 \mathrm{GHz}$. The broken lines do not include noise while the solid lines are calculated when a spontaneous emission noise term (Langevin noise source) is included in the photon rate equation [8]. Noise terms in the equations for carrier density are neglected since it is known that these terms are less important in predicting the properties of the laser [8]. 
First, we consider the case where the noise term is neglected. When the applied optical signal is removed, the SPLD returns to its free-running frequency within a couple of pulses, as was shown in Fig. 2. When the SPLD has reverted to its freerunning frequency, a constant phase drift is introduced to each pulse due to the difference in frequencies. It is simple to show this phase drift per pulse is given by

$$
\Delta \Phi_{\text {drift }}=\left\{360^{\circ} \times\left[1-\frac{f_{F R}}{f_{\text {app }}}\right]\right\} .
$$

For a free-running frequency of $1.99 \mathrm{GHz}$ and a synchronizing signal frequency of $2 \mathrm{GHz}$, the data signal and the clock would be $90^{\circ}$ out of phase after 50 pulses. As the difference between the free-running and synchronizing signals is decreased, the less the phase slip.

Theoretically, for a no-noise situation, if the two frequencies were exactly equal, the clock and data would remain synchronized regardless of the number of zeros in succession. Therefore, to reduce the read errors in the system, the SPLD free-running frequency should be as close as possible to the expected data signal frequency. This highlights the need for a high-power data signal, because the closer the free-running SPLD frequency and the applied signal frequency, then the longer the laser takes to synchronize (see Fig. 2). If, after the train of 0 's, a 1 is received, this would attempt to pull the SPLD back into synchronization; a high-power data signal helps because fewer 1's are need to fully resynchronize the laser.

For the realistic case where spontaneous emission noise is included in the model, the evolution of the instantaneous phase is given by the solid lines. As expected, the same general trend is seen but the pulse-to-pulse jitter is evident. As discussed in a previous paper [8], when an SPLD is synchronized to an applied signal, the pulse-to-pulse jitter is unaffected but the longterm accumulation of timing jitter is reduced. When the synchronizing signal is removed, the longterm jitter accumulated means that even when the frequency difference between the free-running SPLD and the data signal is minimal a read error could eventually occur. However, as shown in Fig. 9, the magnitude of the noise means that the number of 0's that could be accommodated is reasonably large.

\section{CONCLUSIONS}

We have investigated the dynamics of a self-pulsating laser diode under the application of an external synchronizing signal. In particular, we determine the time it takes for the laser to assume the same frequency as the input signal and also how soon it reverts back to its free-running frequency when the signal is removed. Increasing the power of the input signal is shown to reduce the time it takes for the laser to lock to the synchronizing signal which is desirable to reduce errors in clock extraction systems. We also identify a problem with achieving lock when the input signal is too powerful, and this must be considered when optimizing the operating signal power. The phase difference which exists between the input signal and the output pulse is dependent on the input power and frequency detuning, and the effect this would have on a clock extraction subsystem is outlined. Finally, we show how errors would be introduced when the laser is synchronized using a true data signal by calculating the drift of the phase difference between the signal and the clock obtained when the input signal is removed to simulate 0 bits.

\section{REFERENCES}

[1] P. E. Barnsley, G. E. Wickens, H. J. Wickes, and D. M. Spirit, "All-optical clock recovery from a $5 \mathrm{~GB} / \mathrm{s}$ RZ data signal using a selfpulsating $1.56 \mu \mathrm{m}$ laser diode," IEEE Photon. Technol. Lett., vol. 3, pp. 942-945, Oct. 1991.

[2] G. Farrell, P. Phelan, J. Hegarty, and J. Shields, "All-optical timing extraction with frequency division using a twin section laser diode," IEEE Photon. Technol. Lett., vol. 5, pp. 718-721, June 1993.

[3] A. Valle, P. McEvoy, P. Rees, L. Pesquera, J. O'Gorman, and P. Phelan, "Bit error rate analysis of optical clock extraction using self pulsating laser diodes," IEEE J. Quantum Electron., submitted for publication.

[4] M. Yamada, "A theoretical analysis of self-sustained pulsation phenomena in narrow-stripe semiconductor lasers," IEEE J. Quantum Electron., vol. 29, pp. 1330-1336, May 1993.

[5] A. Egan, M. Harley-Stead, P. Rees, S. Lynch, P. McEvoy, J. O'Gorman, and J. Hegarty, "All-optical synchronization of self-pulsating laser diodes," Appl. Phys. Lett., vol. 68, no. 25, pp. 3534-3536, 1996.

[6] E. A. Avrutin, "Analysis of spontaneous emission and noise in selfpulsing laser diodes," Proc. Inst. Elect. Eng., pt. J, vol. 140, no. 1, pp. 16-20, 1993.

[7] A. Egan, M. Harley-Stead, P. Rees, S. Lynch, P. McEvoy, J. O’Gorman, and J. Hegarty, "All-optical synchronization of self-pulsating laser diodes with frequency multiplication and division," J. Mod. Opt., vol. 45, no. 6, pp. 1239-1248, 1998.

[8] A. Egan, M. Harley-Stead, P. Rees, S. Lynch, J. O'Gorman and J. Hegarty, "An experimental and theoretical analysis of jitter in selfpulsating lasers synchronised to periodic electrical signals," IEEE Photon. Technol. Lett., vol. 8, pp. 758-760, June 1996.

P. Rees, photograph and biography not available at the time of publication.

P. McEvoy, photograph and biography not available at the time of publication.

A. Valle, photograph and biography not available at the time of publication.

J. O'Gorman, photograph and biography not available at the time of publication.

S. Lynch, photograph and biography not available at the time of publication.

P. Landais, photograph and biography not available at the time of publication.

L. Pesquera, photograph and biography not available at the time of publication.

J. Hegarty, photograph and biography not available at the time of publication. 